\title{
Orientation specificity of length assimilation and contrast
}

\author{
KEVIN JORDAN and JERAYR HALEBLIAN \\ San Jose State University, San Jose, California
}

\begin{abstract}
The orientation specificity of length assimilation and contrast was assessed using the parallellines array. The purpose was to determine the nature of the representation of length both within and outside of the "attentive field." Experiments 1 and 2 demonstrated that when both contextual and test lines are within an attentive field and do not intersect, the resulting simultaneous length assimilation is independent of orientation. In Experiment 3, simultaneous length contrast was produced by placing the contextual line outside of the attentive field; it also was independent of orientation. Finally, Experiment 4 demonstrated that the sequential length contrast (length aftereffect) produced by a 5-sec prior inspection of a contextual line was independent of orientation, whereas the contrast produced by $60 \mathrm{sec}$ of contextual-line inspection showed some degree of orientation specificity. These results, combined with those obtained with orientation illusions (Tyler \& Nakayama, 1984; Wenderoth \& Johnson, 1984; Wenderoth, O'Connor, \& Johnson, 1986), suggest a qualitative relationship of length and orientation coding that is characterized by partial and mutual overlap of length and orientation information.
\end{abstract}

The parallel-lines array is a minimal configuration for producing an illusion of perceived length. The direction of this distortion of perceived length depends on the spatial and temporal relationship of the test line and a parallel contextual line. In the usual parallel-lines array, the contextual and test lines are presented simultaneously and spatially proximal, and length assimilation is observed. For example, at a viewing distance of $40 \mathrm{~cm}$, a $9-\mathrm{cm}$ horizontal contextual line will produce overestimation of a parallel 6-cm test line located $5 \mathrm{~mm}$ below it. However, length contrast has been demonstrated in the array under conditions of simultaneous viewing with a much larger spatial separation $(100 \mathrm{~mm})$ of the same contextual and test lines (Jordan \& Schiano, 1986) and under conditions of sequential presentation of contextual and test lines within the aftereffect paradigm (Brigell \& Uhlarik, 1979; Jordan \& Uhlarik, 1985).

Jordan and Schiano (1986) proposed revisions of descriptive models of length distortion that account for the reversals from length assimilation to length contrast outlined above. Their model is essentially a hybrid of Girgus and Coren's (1982) "pool and store" model of perceptual distortion and the most recent revision of Pressey's assimilation theory (Pressey \& Wilson, 1980). According to Girgus and Coren's model, the visual information-processing system imposes constraints on the amount or the area of an array that an observer can sample simultaneously. The lengths of spatially proximal elements in an array are perceptually "pooled," resulting

Preparation of this report was supported by NASA Grant NCC-2-327 to San Jose State University, Kevin Jordan, Project Director. Jerayr Haleblian is now at the University of Southern California. The authors are pleased to acknowledge the helpful comments of two anonymous reviewers. Requests for reprints should be sent to Kevin Jordan, Department of Psychology, San Jose State University, San Jose, CA 95192. in length assimilation. The lengths of spatially or temporally separated elements in an array are sampled sequentially, leading to the encoding of differences in length, with contrast the result. However, Jordan and Schiano's (1986) model of assimilation and contrast departs from the pool-and-store model in defining the area within which the perceptual pooling of length occurs. Here, they propose that Pressey's (1972) construct of a roughly circular attentive field within which assimilation occurs describes their data. The size of this attentive field is proposed to be a constant ratio of target-stimulus size (Pressey \& Di Lollo, 1978). In the specific case of the parallellines array, Jordan and Schiano propose that (1) the attentive field is circular, (2) the radius of the attentive field is the test-line length, and (3) the attentive field is centered in the middle of the test line. When contextual and test stimuli in an array fall within the attentive field (e.g., when presented simultaneously and spatially proximally), assimilation results. On the other hand, contrast results when the test stimulus is within the attentive field (which is true by definition, since the center of the test line is proposed to be the center of the attentive field) while the contextual elements are outside of the attentive field. This effect is due to either a large spatial separation or to sequential presentation, as in the aftereffect paradigm.

Our research is currently directed at investigating the nature of the representations of length inside and outside of the attentive field. We propose that these representations are pooled and stored, respectively. Thus, we seek to determine whether length information is coded independently or whether it is coded along with other parameters of form, such as orientation. Figure 1 presents five possible relationships of length $(L)$ and orientation $(O)$ coding in the visual system (based on Virsu \& Haapasalo, 1973, who presented a similar treatment of color and 
A.
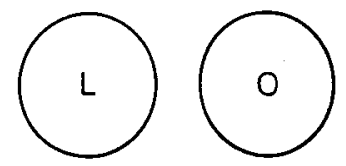

B.

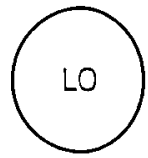

C.

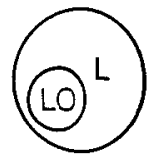

D.

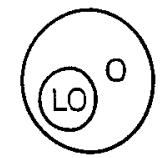

E.

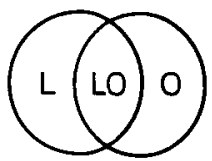

Figure 1. Five possible relationships between length $(L)$ and orientation $(O)$ coding. $A$, length and orientation are coded separately; $B$, length and orientation coding completely overlap; $C$, orientation is coded as a subset of length; $D$, length is coded as a subset of orientation; $E$, length and orientation coding partially overlap (adapted from Virsu \& Haapasalo, 1973).

spatial-frequency coding). In relationship A, length and orientation information are coded independently, whereas in relationship B, there is a complete overlap or redundancy of length and orientation coding. relationships $\mathrm{C}-\mathrm{E}$ represent the possibilities of partial overlap of length and orientation coding. This set of qualitative relationships makes no assumptions or assertions regarding the physical structures underlying the representation of length and orientation.

There are data available that allow some choices to be made among the qualitative relationships of length and orientation coding presented in Figure 1. For example, Wenderoth, O'Connor, and Johnson (1986) recently reported that the magnitude of simultaneous orientation contrast (the tilt illusion) was a function of the length of the test and inducing lines. To summarize, they reported changes in the magnitude of the tilt illusion when the length of the test (or contextual) arm of an angle was held constant while the contextual (or test) arm length was varied (see Wenderoth et al., 1986, Figure 3). Additionally, Tyler and Nakayama (1984) reported that the magnitude of simultaneous orientation assimilation and contrast in a variant of the Zollner-illusion configuration was contingent on the length of the individual line segments comprising the figure. These results indicate some overlap of orientation and length coding within the mechanisms that produce the orientation distortions. Thus, rela- tionship $\mathrm{A}$ is not a correct description of length and orientation coding within the visual system. Additionally, Wenderoth and Johnson (1984) reported that the magnitude of the tilt illusion was not changed when the length of both the contextual and test arms forming an angle varied. This result indicates at least a partial independence of orientation and length coding, and thus rules out relationships B (redundancy of coding) and C (orientation coding as a subset of length coding). Relationships $\mathrm{D}$ and $\mathrm{E}$ remain as potential descriptions of the relationship between orientation and length coding. In order to test between these two relationships, the orientation specificity of length distortions must be examined. If length distortions are shown to depend on the orientation of contextual and test stimuli under all conditions, then relationship D would be indicated. However, if length distortions can be shown to be independent of orientation under some conditions, then relationship $\mathrm{E}$ would be indicated.

The present paper reports on a series of experiments that investigated the orientation specificity of length assimilation and contrast within the illusion and aftereffect paradigms. Experiments 1 and 2 tested for orientation specificity of simultaneous length assimilation, whereas Experiment 3 tested for orientation specificity of simultaneous length contrast. Finally, Experiment 4 examined the orientation specificity of sequential length contrast.

\section{EXPERIMENT 1}

In the first experiment, we examined the orientation specificity of simultaneous length assimilation (the usual parallel-lines illusion). The orientation of the contextual line in the parallel-lines array was manipulated so that it was no longer parallel to the test line; the effects on assimilation magnitude were then assessed. Four levels of orientation of the contextual line relative to that of the test line were used: $0^{\circ}$ (the parallel-lines array), $12^{\circ}, 45^{\circ}$, and $90^{\circ}$. If length information interacts with orientation information as suggested by relationship $D$, the magnitude of assimilation of test-line length would be expected to vary with the orientation of the contextual line.

\section{Method}

Observers. Eighteen members of an introductory psychology course at San Jose State University participated in the experiment in order to earn course credit. All observers were run in individual sessions.

Stimuli and Design. All stimulus lines were made with black tape on a white background. They were then photographed, and the resulting 35-mm slides were projected on the rear of a translucent screen. The order and duration of stimulus presentation were controlled by a programmable Bell and Howell random-access projector (Model 850A).

The lengths of the contextual and test lines were chosen to maximize length assimilation prior to the manipulation of orientation. One general finding with length assimilation in the parallel-lines array is that the overestimation produced by contextual lines longer than the test line is much greater than the underestimation produced by shorter contextual lines (Brigell \& Uhlarik, 1979; Jordan \& Schiano, 1986; Jordan \& Uhlarik, 1985). Additionally, Brigell and Uhlarik reported that this overestimation was greatest when the ra- 
tio of contextual length to test length (the "framing ratio") was between 1.5 and 2.0 . Based on these findings, the projected contextual length used in the present experiment was $108 \mathrm{~mm}$ and the test length was $72 \mathrm{~mm}$, resulting in a framing ratio of 1.5 . The thickness of the lines on the viewing screen was $1.5 \mathrm{~mm}$, and the viewing distance was $60 \mathrm{~cm}$.

There were four levels of orientation of the contextual line relative to that of the horizontal test line $-0^{\circ}$ (the parallel-lines array), $12^{\circ}, 45^{\circ}$, and $90^{\circ}$. The $12^{\circ}$ orientation was chosen because it produces peak orientation illusion in a $0^{\circ}$ line (e.g., Magnussen \& Kurtenbach, 1980) and thus is thought to be within the bandwidth of a central neural response function for a $0^{\circ}$ line. The $45^{\circ}$ condition was chosen because this oblique orientation usually does not affect responses to a $0^{\circ}$ line (Magnussen \& Kurtenbach, 1980), and it seems to be the orientation to which observers are least sensitive (Appelle, 1972) and slowest to respond (Attneave \& Olson, 1967). Finally, $90^{\circ}$ was chosen on the basis of reports that distortions of form can be induced by contextual stimuli at orthogonal orientations. For example, Heeley (1979) demonstrated a shift in the perceived spatial frequency of a sinusoidal test grating induced by an orthogonally oriented adapting grating.

In the $0^{\circ}$ condition of the present experiment, the contextual line was parallel to, and centered $12.5 \mathrm{~mm}$ above, a horizontally oriented test line. The orientation of the contextual line was manipulated by rotating the contextual line about its center (see Figure 2). Note that the $45^{\circ}$ and $90^{\circ}$ contextual lines intersect the test line.

Judgments of the test line were obtained with a graded-series comparison scale (cf. Coren \& Girgus, 1972), which was located $20 \mathrm{~cm}$ to the right of the midpoint of the test line on each slide. The scale consisted of 11 horizontally oriented lines, labeled $A$ through $K$, which ranged in length from 60 to $84 \mathrm{~mm}$ in 2.4-mm increments. Thus, the point of objective equality (POE) on this scale was $F$, or $72 \mathrm{~mm}$ (i.e., the scale length that physically matched the test length). The lines on the scale were $1.5 \mathrm{~mm}$ thick, and were separated vertically by $12 \mathrm{~mm}$. To prevent response bias, we encouraged the observers to use a wide range of the response scale by including four filler stimuli in the design. The test lengths for these stimuli ranged from 62.4 to $81.6 \mathrm{~mm}$, and the contextual orientations were randomly determined. Responses to these filler stimuli were not included in the data analysis.

Finally, to familiarize the observers with the use of the response scale, there were also six practice stimuli, which consisted of only
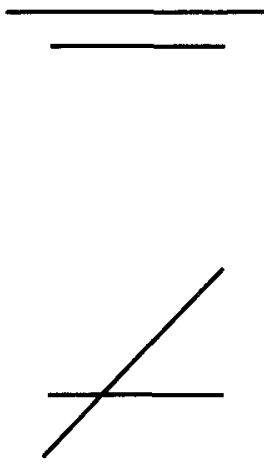

Figure 2. Schematic representation of the four test stimuli in Experiment 1. The horizontal test line was $72 \mathrm{~mm}$ long, whereas the contextual line was $108 \mathrm{~mm}$ long and oriented at (a) $0^{\circ}$, (b) $12^{\circ}$, (c) $45^{\circ}$, or (d) $90^{\circ}$ relative to the test line. The center of the contextual line was $12.5 \mathrm{~mm}$ above the test line. a test line; there was no contextual line. The lengths of the judged line in the practice stimuli were $62.4,67.2,72,72,76.8$, and $81.6 \mathrm{~mm}$.

Procedure. The observers viewed the projected image of the stimuli under normal fluorescent room lighting. The observers were familiarized with the range and variety of the stimuli and with the use of the response scale. Specifically, they were told that the task was to match the apparent length of the test line to one of the 11 lines on the response scale by calling out the letter of the match to the experimenter.

After receiving these instructions, the observers proceeded through a random order of the six practice stimuli, which was followed by a random order of the four test and four filler stimuli. After a 30-sec break, this procedure was repeated with new random orders of the practice, test, and filler stimuli. A trial consisted of a 5-sec presentation of the test or filler slide, during which the test judgment was to be made. The slide contained the contextual and test lines, as well as the graded-series comparison scale in the spatial locations described above. The observers viewed the slide freely during its 5 -sec presentation; they were given no instructions regarding fixation. Trials were separated by $10 \mathrm{sec}$, during which the viewing screen was blank. In all, each of the 18 observers made 28 length judgments: 2 judgments each of the six practice, four test, and four filler stimuli. The entire procedure required approximately $10 \mathrm{~min}$ for each observer.

\section{Results and Discussion}

The responses to the 72-mm no-context lines obtained during the first practice series were used to estimate the point of subjective equality (PSE) of the $72-\mathrm{mm}$ line contained in the test stimuli. The mean of the 36 responses to this control line ( 2 responses from each of 18 observers) was $66.5 \mathrm{~mm}$. This underestimation of objective test-line length has been consistently reported in studies that used the graded-series comparison scale (Brigell \& Uhlarik, 1979; Jordan \& Schiano, 1986; Jordan \& Uhlarik, 1985, 1986). Additionally, Coren and Girgus (1978, p. 179) pointed out that this psychophysical "error of the standard" is often reported when observers attempt to match a standard length to a variable set of lines.

Judgments of the 72-mm line contained in the test stimuli were converted into deviations from PSE by subtracting the PSE from the mean judged length for each test stimulus. These data are presented in Figure 3. First, note the substantial overestimation of the $72-\mathrm{mm}$ test line produced by the parallel 108-mm contextual line (see the data point for the $0^{\circ}$ orientation of the contextual line in Figure 3 ). This is simply a demonstration of simultaneous length assimilation in the parallel-lines array. There was also substantial length assimilation when the contextual line was oriented at $12^{\circ}$ relative to the test line. Finally, there was little evidence of assimilation for the $45^{\circ}$ and $90^{\circ}$ conditions.

The data were analyzed in a 4 (orientations) $\times 2$ (replications) $\times 18$ (observers) within-subjects analysis of variance. The main effect of orientation was statistically significant $[F(3,51)=17.17, p<.01]$, indicating that the orientation of the contextual line affected test-line judgments differentially. The direction of this effect is a decrease in assimilation magnitude as the contextual-line orientation changes relative to the test-line orientation. There was also a significant main effect of replications 
$[F(1,17)=13.17]$, which indicated that the responses in the second replication $(M=69.6 \mathrm{~mm})$ of the test stimuli were greater than the responses in the first replication $(M$ $=67.9 \mathrm{~mm}$ ). However, replications did not interact with orientation $[F(3,51)<1]$, so the data are pooled across replications in Figure 3. Planned comparisons indicated that the judgments for the $0^{\circ}$ and $12^{\circ}$ conditions did not differ from each other, but they did differ from the $45^{\circ}$ and $90^{\circ}$ conditions. These latter conditions did not differ significantly from the PSE.

A reasonable interpretation of these data would be that they demonstrate an orientation-contingent line-length illusion. However, the presence of the length illusion was also contingent on the intersection of the contextual and test lines. In the $0^{\circ}$ and $12^{\circ}$ conditions, there was a significant illusion and the lines did not intersect; in the $45^{\circ}$ and $90^{\circ}$ conditions, there was no illusion and the lines did intersect. Thus, orientation was confounded with intersection. This is an important consideration since line bisection per se is known to produce the visual illusion of underestimation (Coren \& Girgus, 1978), and this underestimation might have counteracted, or canceled, any assimilation (overestimation) in the $45^{\circ}$ and $90^{\circ}$ conditions. Experiment 2 removed this confound of orientation and line intersection.

\section{EXPERIMENT 2}

The present experiment, like Experiment 1, tested for orientation specificity of simultaneous length assimilation. However, the confound of orientation of the contextual line and intersection of the test line was removed by shift-

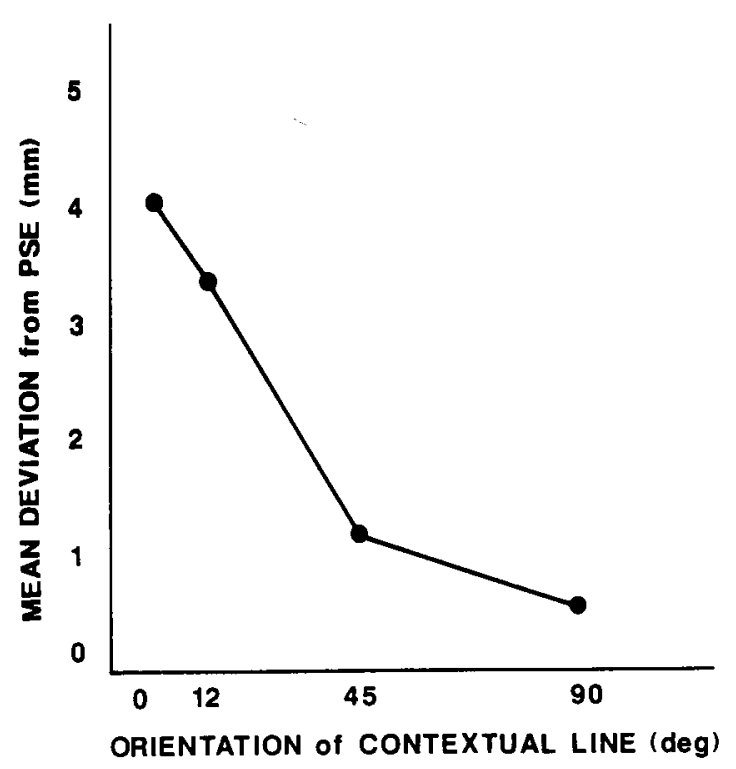

Figure 3. Mean deviations from the point of subjective equality (PSE), in millimeters, for the four orientation conditions of Experiment 1 . The overestimation of the $72-\mathrm{mm}$ test line produced by the $108-\mathrm{mm}$ contextual line in the $0^{\circ}$ condition is a demonstration of simultaneous length assimilation (the parallel-lines illusion).

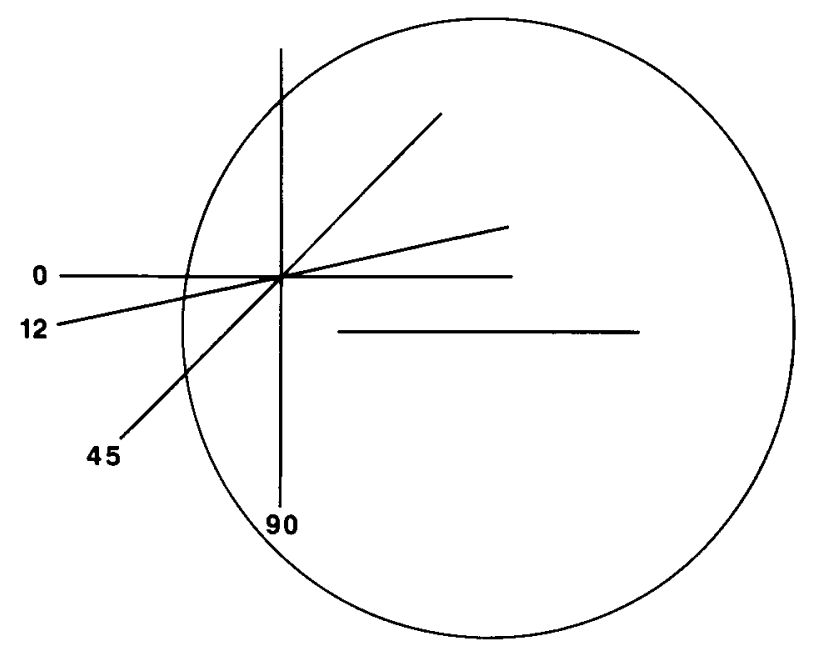

Figure 4. Schematic representation of the four test stimuli in Experiment 2. The center of the contextual line was shifted $50 \mathrm{~mm}$ to the left of its position in Experiment 1 (see Figure 2). All other stimulus parameters remained the same as those in Experiment 1. The circle represents the attentive field with a radius equal to the length of the test line.

ing the position of the contextual line relative to the test line. Figure 4 presents a schematic of this manipulation. The center of the parallel $\left(0^{\circ}\right)$ contextual line was shifted $50 \mathrm{~mm}$ to the left and orientation was manipulated by rotating the contextual line about its center. The net result was a manipulation of contextual-line orientation without any intersection of the test line.

In attempting to anticipate the results, it is important to note that parts of the contextual line now fall outside of the attentive field. The circle in Figure 4 represents the attentive field boundary based on the Jordan and Schiano (1986) model: the attentive field is centered at the middle of the test line, and its radius is the test-line length. Given this arrangement, the $0^{\circ}, 12^{\circ}$, and $45^{\circ}$ contextual lines fall partially outside the attentive field with proportionately more of the $45^{\circ}$ line within the attentive field. The $90^{\circ}$ contextual line falls almost entirely within the attentive field. If assimilation is only produced by contextual stimuli within the attentive field, then progressively reduced assimilation would be expected in the $0^{\circ}$ and $12^{\circ}$ conditions relative to Experiment 1 . This should occur because the part of the contextual line remaining in the attentive field is similar in length to the test line, and lines of similar length do not affect one another (Brigell \& Uhlarik, 1979). However, since the $90^{\circ}$ contextual line is within the attentive field, and since the confound with intersection has been removed, we now have a better test of the ability of the $90^{\circ}$ contextual line, and, to a lesser extent, the $45^{\circ}$ line, to produce simultaneous length assimilation.

\section{Method}

Observers. A new group of 18 members of an introductory psychology course participated in the experiment in order to earn course credit. The observers were again run in individual sessions. 
Stimuli and Design. The stimuli and design of this experiment were identical to those of Experiment 1 in all respects except for the location of the contextual line relative to the test line. The parallel $\left(0^{\circ}\right)$ contextual line was still $12.5 \mathrm{~mm}$ above the horizontal test line, but it was shifted $50 \mathrm{~mm}$ to the left. Orientation was again manipulated by rotating the contextual line about its center (see Figure 4).

Procedure. The procedure was identical in all respects to that of Experiment 1.

\section{Results and Discussion}

The PSE, which was estimated in the same manner as in the previous experiment, was $67.9 \mathrm{~mm}$. This underestimation of objective test-line length was also observed in Experiment 1, as well as in our prior research. Mean deviations from PSE were obtained by subtracting this PSE from the mean judged length for each of the four test stimuli. These data are presented in Figure 5. First, note that there is very little length assimilation in the $0^{\circ}$ and $12^{\circ}$ conditions. This may be due to the contextual line's being partially outside the attentive field in these two conditions (see Figure 4). However, there is substantial length assimilation in the $45^{\circ}$ and $90^{\circ}$ conditions, which was not evident for these same orientations in Experiment 1 .

The data were analyzed in a 4 (orientations) $\times 2$ (replications) $\times 18$ (observers) within-subjects analysis of variance. The main effect of orientation was statistically significant $[F(3,51)=12.58, p<.01]$, indicating that contextual lines influenced the test-line length judgments differentially. The direction of this effect, however, is opposite of the effect in Experiment 1. Once again, the main effect of replications was significant, but this factor did not interact with orientation. Therefore, the data are pooled over replications in Figure 5. Planned comparisons indicated that the magnitude of length assimilation

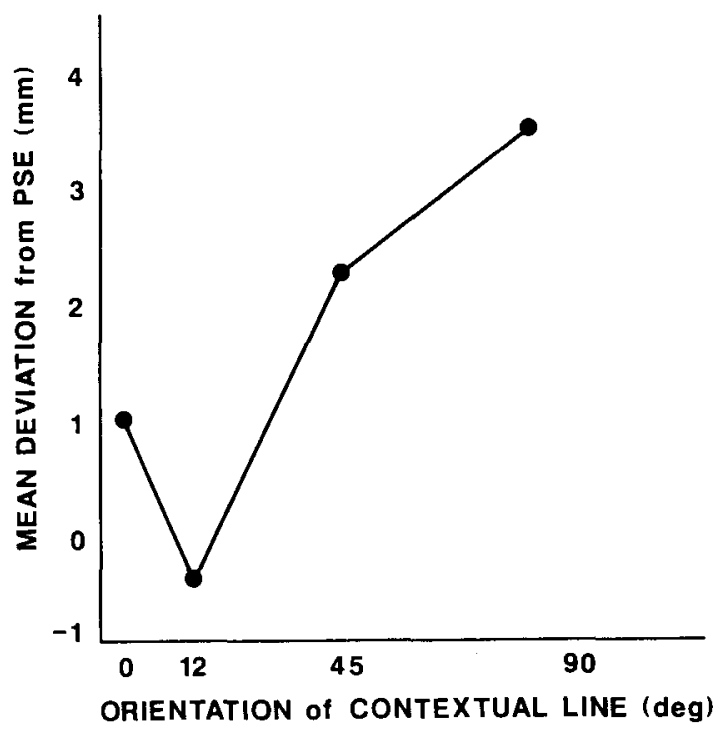

Figure 5. Mean deviations from the point of subjective equality (PSE), in millimeters, for the four orientation conditions of Experiment 2. did not differ significantly for the $45^{\circ}$ and $90^{\circ}$ conditions, but did differ for the $0^{\circ}$ and $12^{\circ}$ conditions. These latter conditions did not differ significantly from the PSE.

An interesting result of Experiment 2 is that the simple shift of the parallel contextual line relative to the test line $\left(0^{\circ}\right.$ condition) abolished the illusion. Brigell and Uhlarik (1979) argued that the framing ratio determined the magnitude of the parallel-lines illusion. However, the framing ratios were identical for the $0^{\circ}$ conditions of Experiments 1 and 2 , yet the illusion was present in one case (Experiment 1) but not in the other (Experiment 2). These results argue against framing ratio as the predictor of illusion magnitude. It seems that the predictor of assimilation magnitude in this case was the amount of the contextual line falling within the attentive field; the greater the contextual line's extent within the attentive field, the greater the assimilation magnitude (the "range" postulate, Pressey \& Murray, 1976). Contextual line extents outside of the attentive field are ineffective in producing assimilation and may produce contrast (Pressey \& Wilson, 1980). Thus, as the contextual line was shifted $50 \mathrm{~mm}$ to the left in Experiment 2, part of the contextual line in the $0^{\circ}$ condition fell outside of the attentive field, and there was effectively less of the contextual line within the attentive field. Pressey and Smith (1986) reported a similar result with the Baldwin-illusion figure, which contains a horizontal test line flanked by two contextual boxes. They moved the contextual boxes so that they were both on the same end of the test line, and the assimilation illusion was significantly reduced. As in our experiments, the framing ratio remained constant but illusion magnitude changed. We argue that the reduced magnitude of the Baldwin illusion reported by Pressey and Smith occurred for the same reason as the reduced magnitude of the parallel-lines illusion in our Experiment 2; part of the context (a box) fell outside of the attentive field, and there was less efective context remaining within the attentive field to produce assimilation.

A summary description of the combined results of Experiments 1 and 2 would be that when a contextual line is within the attentive field defined by the test line and does not intersect the test line, it can produce length assimilation regardless of its orientation. This seems to describe the data for the $0^{\circ}$ and $12^{\circ}$ conditions of Experiment 1 and the $90^{\circ}$ condition of Experiment 2. Given the assumption of the model that the radius of the attentive field is the test length, it is not clear why assimilation occurred in the $45^{\circ}$ condition of Experiment 2. One possibility is that the radius of the field is somewhat larger than the test length, which would result in the $45^{\circ}$ line's being almost entirely within the attentive field. More precise studies of the boundaries and the shape of the attentive field are clearly needed. An alternative account of the assimilation observed in the $45^{\circ}$ condition is that the effect is simply a variant of the Ponzo illusion. That is, the $45^{\circ}$ contextual line can be thought of as one of the two oblique inducing lines in the usual Ponzo-illusion configuration. Fisher (1968) showed that the Ponzo line- 
length illusion occurred with only one of the oblique inducing lines present, although its magnitude was reduced. Recently, Jordan and Randall (1987) presented a revised assimilation-theory account of the Ponzo illusion that predicts both the assimilation effect observed in the $45^{\circ}$ condition of the present experiment and its reduced magnitude relative to the $90^{\circ}$ condition.

In terms of the Jordan and Schiano (1986) model of assimilation and contrast, the data of Experiments 1 and 2 indicate that the representation of length within the attentive field is independent of orientation. Put another way, the representations of length that are pooled to produce assimilation within this attentive field are orientation-free. Rreferring to the analysis described in Figure 1, the results of Tyler and Nakayama (1984) and Wenderoth et al. (1986) rule out relationship A. Additionally, the results of Wenderoth and Johnson (1984) rule out relationships B and C. Finally, the results of these first two experiments rule out relationship $\mathrm{D}$, since the obtained length distortions seemed independent of orientation. Thus, within the attentive field, length and orientation coding seem to partially and mutually overlap (relationship E). However, this conclusion must be qualified due to the inevitable confounding of the orientation manipulation with either the bisected-line illusion (Experiment 1) or the Ponzo illusion (Experiment 2).

The remaining experiments examine the nature of the relationship between length and orientation coding when the contextual line is either spatially or temporally outside the attentive field produced by the test line-that is, under the conditions that produce length contrast.

\section{EXPERIMENT 3}

Jordan and Schiano (1986) first reported simultaneous length contrast in the parallel-lines figure. Simultaneous length assimilation reversed to simultaneous length contrast with large spatial separation of the contextual and test lines. Importantly, the amount of spatial separation needed for the reversal depended on test-line length. Jordan and Schiano reported that contrast resulted whenever parallel contextual and test lines were separated perpendicularly by more than the test length. According to their model, the contextual line was then outside of the attentive field, leading to sequential sampling of the contextual and test lines. In the present experiment, the center of the contextual line was $125 \mathrm{~mm}$ above the horizontal 72-mm test line. The orientation of the contextual line was then varied and the effects on contrast magnitude were assessed in order to test for the orientation specificity of simultaneous length contrast.

\section{Method}

Observers. A new group of 18 introductory psychology students participated in the experiment. Again, all observers were run in individual sessions.

Stimuli and Design. The stimuli and design of this experiment were identical to those of Experiment 1 except for the length and the relative position of the contextual line.
The length of the contextual line was again chosen to maximize length contrast prior to the manipulation of orientation. As with length assimilation, the overestimation of test-line length is much greater than underestimation in length-contrast effects (Brigell \& Uhlarik, 1979; Jordan \& Schiano, 1986; Jordan \& Uhlarik, 1985). However, in length contrast, overestimation of test-line length is produced by contextual lines shorter than the test line (this is the definition of length contrast). Additionally, Brigell and Uhlarik reported that peak length contrast was produced when the ratio of contextual to test length (the framing ratio) was about 0.67 . Based on these considerations, the contextual length in the remaining experiments on length contrast was $48 \mathrm{~mm}$ and the test length was $72 \mathrm{~mm}$ (framing ratio $=0.67$ ). Although consistency of contextual length across experiments would normally be desirable, it should be emphasized that we had to change the length of the contextual line in this experiment; if we had used the 108 -cm contextual line, there would have been no length contrast (see Jordan \& Schiano, 1986, Figure 2).

There were again four levels of orientation of the contextual line relative to the test line $-0^{\circ}, 12^{\circ}, 45^{\circ}$, and $90^{\circ}$. In the $0^{\circ}$ condition, the $48-\mathrm{mm}$ contextual line was parallel to, and centered $125 \mathrm{~mm}$ above, the horizontal $72-\mathrm{mm}$ test line. The orientation of the contextual line was again manipulated by rotating the contextual line about its center.

Procedure. The procedure was identical in all respects to that of the previous experiments.

\section{Results and Discussion}

The PSE, which was estimated in the same manner as in the previous experiments, was $67 \mathrm{~mm}$, which is consistent with the underestimation of objective length observed in Experiments 1 and 2. Mean deviations from PSE were obtained by subtracting this PSE from the mean judged length for each of the four test stimuli. These data are presented in Figure 6; they indicate the presence of

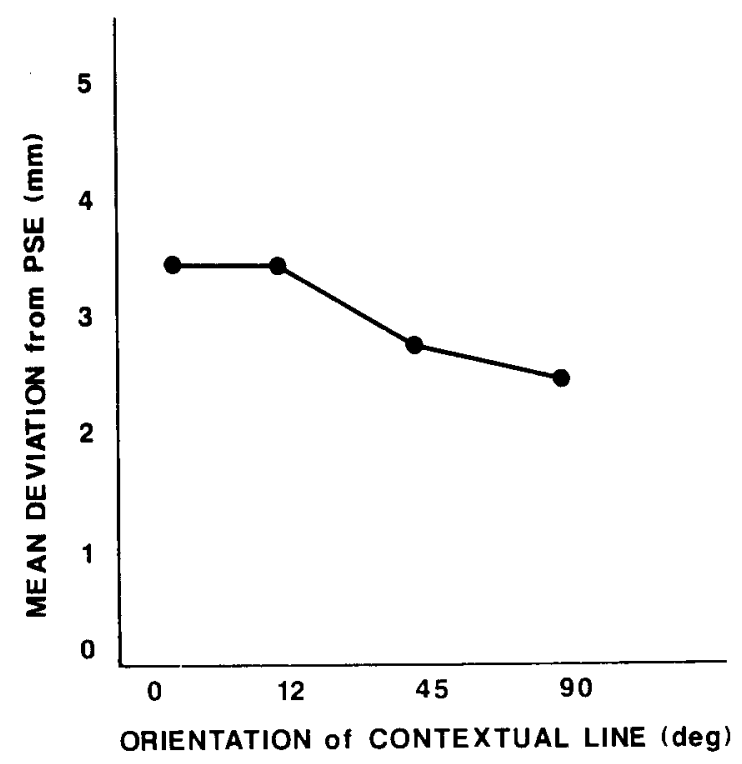

Figure 6. Mean deviations from the point of subjective equality (PSE), in millimeters, for the four orientation conditions of Experiment 3. The overestimation of the 72-mm test line produced by the 48-mm contextual line in the $0^{\circ}$ condition is a replication of Jordan and Schiano's (1986) initial demonstration of simultaneous length contrast in the parallel-lines figure. 
length contrast. For example, in the $0^{\circ}$ condition, a 48$\mathrm{mm}$ contextual line that was parallel to, and centered $125 \mathrm{~mm}$ above, a 72-mm horizontal test line produced overestimation of that test line. This simultaneous lengthcontrast effect replicates Jordan and Schiano's (1986) initial report of the reversal from assimilation to contrast in the parallel-lines array produced by a large spatial separation of contextual and test lines. Note also the substantial length contrast observed at the other three orientations of the contextual line.

The data were analyzed in a 4 (orientations) $\times 2$ (replications) $\times 18$ (observers) within-subjects analysis of variance. The main effect of orientation did not reach statistical significance $[F(3,51)=1.76, p>.05]$, indicating that contextual-line orientation did not affect testline-length judgments differentially. However, planned comparisons indicated that the judgments at all orientations were significantly different from the PSE. The main effect of replications was again significant, but replications did not interact with orientations. Therefore, the data are pooled over replications in Figure 6.

The results of the statistical analyses indicate that the manipulation of the spatial separation of contextual and test lines produced length contrast, and that this length contrast was not specific to the orientation of the contextual line. In the context of the Jordan and Schiano (1986) model of assimilation and contrast, the data indicate that length is coded outside of the attentive field independently of its orientation, and thus that our stored representation of length is orientation-free. Compared to the conclusions based on the data of Experiments 1 and 2, this conclusion indicates a qualitative similarity of length coding within and outside of the attentive field. Additionally, the present data support the conclusion of Experiments 1 and 2 that relationship E (mutual and partial overlap) is the correct description of the relationship of length and orientation coding in the visual system. The final experiment examined whether these conclusions generalize to sequential length contrast produced within an aftereffect paradigm.

\section{EXPERIMENT 4}

This final experiment examined the orientation specificity of sequential length contrast using the aftereffect paradigm. Brigell and Uhlarik (1979) first reported that a 60-sec inspection of a contextual line produced length contrast in a subsequently judged test line. Additionally, Jordan and Uhlarik (1985) reported that this length aftereffect could be induced by only $5 \mathrm{sec}$ of prior inspection of a contextual line. This result was important because it indicated that temporal separation, rather then prolonged inspection of the contextual line, was responsible for the shift from length assimilation to length contrast. Girgus and Coren's (1982) pool-and-store model was explicit in proposing a functional equivalence of spatial and temporal separation of the contextual and test lines in producing length contrast; this part of their model is included in the
Jordan and Schiano (1986) model. Either kind of separation would require sequential sampling of contextual and test lines, since in both cases the contextual stimulus would be outside of the attentive field produced by the test line.

In the previous experiment, spatial separation of the contextual and test lines produced length contrast, and this contrast did not depend on the orientation of the contextual line. If temporal separation produces length contrast through the same storing process as spatial separation, as suggested by Girgus and Coren (1982), then it would be expected that the length contrast produced within an aftereffect paradigm would not depend on the orientation of the contextual line as well. This expectation is especially strong for an aftereffect condition in which the contextual line is inspected for $5 \mathrm{sec}$ prior to the judgment of the test line, since the time parameters are so similar for this procedure and for the simultaneous length contrast procedure of the previous experiment. In Experiment 3 , the observers saw the contextual and test lines simultaneously and for $5 \mathrm{sec}$. In the present experiment, the observers judged the length of the test line subsequent to either a 5-sec or a $60-\mathrm{sec}$ inspection of a contextual line. For the 5-sec condition, the observers saw both contextual and test stimuli for $5 \mathrm{sec}$, as in Experiment 3, but in the present experiment, the stimuli were presented sequentially.

\section{Method}

Observers. A new group of 36 introductory psychology students participated in the experiment. Eighteen observers were assigned to a 5-sec aftereffect condition, and 18 were assigned to a 60 -sec condition. Again, all observers were run in individual sessions.

Stimuli and Design. The stimuli were constructed, photographed, and projected in the same manner as those in the previous experiments.

The lengths of the contextual and test lines were the same as those in the previous experiment -48 and $72 \mathrm{~mm}$, respectively-in order to maximize length contrast prior to the manipulation of contextualline orientation (see Brigell \& Uhlarik, 1979). The adapting array used in both inspection-duration conditions contained only the 48-mm contextual line, which was centered $60 \mathrm{~mm}$ above the center of the viewing screen. There were again four levels of contextualline orientation relative to the horizontal test line $-0^{\circ}, 12^{\circ}, 45^{\circ}$, and $90^{\circ}$. The orientation of the contextual line was manipulated by rotating the contextual line about its center. The test array contained the 72-mm test line and the graded-series comparison scale in the same spatial locations as in the previous experiments; there was no contextual line in this array.

Judgments of test-line length were obtained using the same gradedseries scale as in the previous experiments. Again, four filler stimuli were included in order to prevent response bias. These filler stimuli involved the same contextual arrays described above, but the test array for these stimuli contained test lines ranging from 62.4 to $81.6 \mathrm{~mm}$. Again, responses to these filler stimuli were not included in the data analysis.

Finally, there were again six practice stimuli, which consisted of only the test line and the graded-series scale. The lengths of the lines in these six stimuli were $62.4,67.2,72,72,76.8$, and $81.6 \mathrm{~mm}$.

Procedure. The observers viewed the projected image of the stimuli under normal fluorescent room lighting. They were given the same instructions regarding the use of the response scale as were given in the previous experiments. The 18 observers in each of the 
aftereffect conditions first proceeded through a random order of the practice stimuli, which was followed by a random order of the four test-stimulus and four filler-stimulus trials. Following a 30sec break, this procedure was repeated with new random orders of the practice-, test-, and filler-stimulus trials. The practice trials consisted of a 5-sec presentation of the practice stimulus, during which the length judgment was to be made. Practice trials were separated by $10 \mathrm{sec}$, during which the viewing screen was blank. The test and filler trials consisted of a 5- or $60-\mathrm{sec}$ presentation of a slide containing only the contextual line, during which the observers were to scan the contextual line. This inspection period was followed by a 5 -sec presentation of the array containing the test line and the response scale (no contextual line), during which the length judgment was to be made. The interval between contextual and test arrays was $1 \mathrm{sec}$. These trials were separated by $20 \mathrm{sec}$, during which the screen was blank. In all, each of the 36 observers in this experiment made 28 length judgments: two judgments of each of the six practice stimuli, and two judgments in each of the four test and four filler trials. The entire procedure required approximately $16 \mathrm{~min}$ for each of the 18 observers in the 5 -sec condition, and $42 \mathrm{~min}$ for each of the 18 observers in the 60 -sec condition.

\section{Results and Discussion}

The PSE, which was estimated in the same manner as in the previous experiments, was $67.2 \mathrm{~mm}$ for the $18 \mathrm{ob}-$ servers in the 5 -sec condition and $68.8 \mathrm{~mm}$ for the $18 \mathrm{ob}$ servers in the 60 -sec condition. This underestimation of objective test-line length was observed in all four experiments in the present report. For each aftereffect condition, the mean deviations from PSE were obtained by subtracting the PSE from the mean judged length for each of the four orientation conditions. These data are presented in Figure 7, and there appears to be substantial length con-

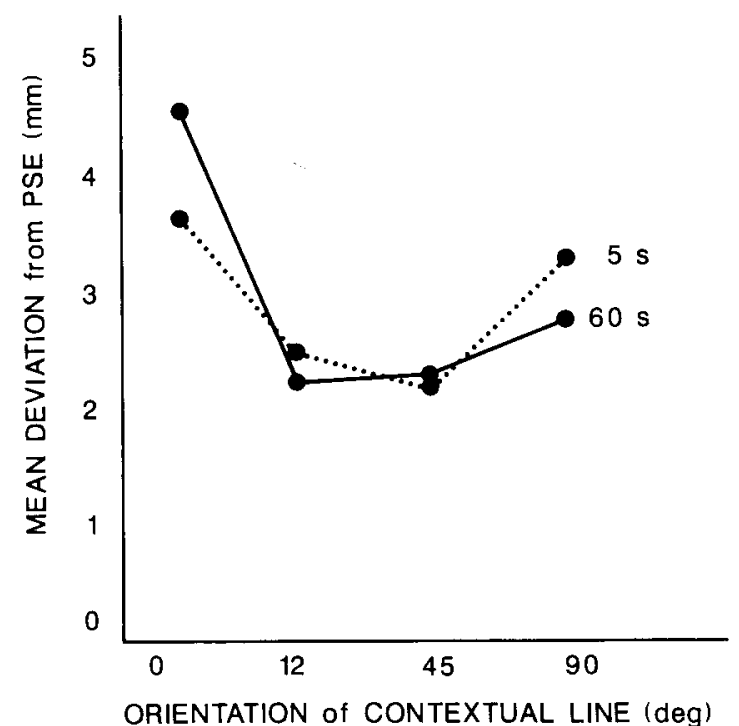

Figure 7. Mean deviations from the point of subjective equality (PSE), in millimeters, for the four orientation conditions and the two contextual-exposure conditions (5 and 60 sec) of Experiment 4. The overestimation of the $72-\mathrm{mm}$ test line in both contextual-exposure conditions produced by the parallel $48-\mathrm{mm}$ contextual line (the $0^{\circ}$ condition) is a demonstration of the parallel-lines aftereffect or sequential length contrast. trast at all orientations for both inspection durations; that is, prior inspection of the $48-\mathrm{mm}$ contextual line resulted in overestimation of the $72-\mathrm{mm}$ test line. Note, however, the apparent orientation specificity in the 60-sec condition, in which the length contrast produced by a 60 -sec inspection of the parallel contextual line $\left(0^{\circ}\right)$ was much greater than the contrast produced at the other three orientations.

The data for each of the inspection conditions were analyzed in separate 4 (orientations) $\times 2$ (replications) $\times$. 18 (observers) within-subjects analyses of variance. For the 5-sec condition, the main effect of orientation did not reach statistical significance $[F(3,51)=1.48, p>.05]$, indicating that contextual-line orientation did not affect test-line length judgments differentially. Planned comparisons indicated that the judgments at all orientations in the 5-sec condition were significantly different from the PSE. Thus, there was length contrast in this condition, but it was not orientation specific. For the 60 -sec condition, the main effect of orientation did reach statistical significance $[F(3,51)=4.56, p<.01]$, indicating that contextual-line orientation affected test-line-length judgments differentially. Planned comparisons indicated that the judgments at all orientations in the 60 -sec condition were significantly different from the PSE. Also, the contrast produced by the $0^{\circ}$ contextual line was greater than the contrast produced at the other orientations. There was length contrast in the $60-\mathrm{sec}$ condition, then, but there was also some degree of orientation specificity in that the greatest amount of contrast was produced when the orientation of the contextual and test lines matched. Neither the main effect of replications nor the interaction of orientation and replications reached statistical significance in either analysis.

The data of the present experiment indicate that under conditions of brief inspection of a contextual line, sequential length contrast is not specific to the orientation of the contextual line, whereas under prolonged inspection conditions, this length aftereffect is somewhat specific to orientation. The data are complementary to the work of Wenderoth and his colleagues, who have reported that under some conditions, orientation contrast is not specific to length (Wenderoth \& Johnson, 1984, Experiment 3) and under other conditions, orientation contrast is lengthspecific (Wenderoth et al., 1986; see also Tyler \& Nakayama, 1984).

In terms of the Jordan and Schiano (1986) model of length assimilation and contrast, the data from this experiment indicate that our stored representation of length (i.e., the representation of length outside the attentive field) is initially orientation-free, but that orientation specificity develops somewhere between 5 and $60 \mathrm{sec}$ of inspection. Despite the first trace of orientation specificity of length distortions in this experiment, the conclusion that the relationship between length and orientation coding in the visual system is one of partial and mutual overlap (relationship $E$ in Figure 1) remains unchanged. In fact, it may be strengthened, in that the exhaustive set of 
possible psychophysical effects that can be derived from this relationship has now been demonstrated: a lengthcontingent orientation distortion (Tyler \& Nakayama, 1984; Wenderoth et al., 1986), a non-length-contingent orientation distortion (Wenderoth \& Johnson, 1984), an orientation-contingent length distortion (Experiment 4, 60-sec condition), and a non-orientation-contingent length distortion (Experiments 1 and 2 combined, Experiment 3, and Experiment 4, 5-sec condition).

\section{GENERAL DISCUSSION}

There were several important results in the present experiments. First, simultaneous length assimilation (the usual parallel-lines illusion) did not seem to be contingent on the orientation of the contextual line relative to that of the test line (Experiments 1 and 2). Simultaneous length contrast also did not seem contingent on contextualline orientation. Finally, sequential length contrast (length aftereffect) induced by $5 \mathrm{sec}$ of exposure to the contextual line prior to the test-line judgment did not seem to be contingent on orientation, where as the aftereffect induced by $60 \mathrm{sec}$ of contextual-line exposure showed some degree of orientation specificity. These findings are discussed in turn as they relate to Jordan and Schiano's (1986) model of length distortion and to the nature of length and orientation coding in the visual system.

\section{Simultaneous Length Assimilation}

The combined results of Experiments 1 and 2 indicated that simultaneous length assimilation was not contingent on the orientation of the contextual line relative to that of the test line. This conclusion must be qualified, however, to state that the orientation of the contextual line does not affect assimilation magnitude as long as the contextual and test lines are within the attentive field and they do not intersect.

According to Jordan and Schiano's (1986) model of length distortion, which is based on Girgus and Coren (1982) and Pressey and Wilson (1980), length assimilation takes place within a roughly circular attentive field (Pressey, 1972) due to the simultaneous sampling (and hence pooling) of the representations of the lengths of contextual and test stimuli. Important departures from the previous models are that the center of the attentive field is the center of the test line, and that the radius of the attentive field is the test length. In Experiment 1, we found that significant length assimilation occurred when the contextual line was either parallel to or oriented $12^{\circ}$ relative to the test line. There was no assimilation when the contextual line was oriented at $45^{\circ}$ or $90^{\circ}$ relative to the test line. However, there was a confound of contextual-line orientation and intersection of contextual and test lines in this experiment. Length assimilation was observed when the two lines did not intersect; it was not observed when the lines did interesect. Given that line intersection leads to underestimation of the line that is intersected (Coren \& Girgus, 1978), it could be that there was length assimilation at $45^{\circ}$ and $90^{\circ}$ but that it was cancelled by this line-intersection illusion.

Experiment 2 removed the confound of contextual-line orientation and intersection of contextual and test lines by shifting the position of the contextual line relative to the test line so that the two lines no longer intersected as orientation was manipulated. Under these conditions, length assimilation was observed at $45^{\circ}$ and $90^{\circ}$ but not at $0^{\circ}$ and $12^{\circ}$. This finding is somewhat consistent with the proposed dimensions of the attentive field. At the $0^{\circ}$, $12^{\circ}$, and, to a lesser extent, $45^{\circ}$ orientations of the contextual line relative to the test line, portions of the contextual line would be outside of an attentive field with the dimensions specified by Jordan and Schiano (1986). As such, contextual-line length within the attentive field would be similar to test-line length, and in this situation, very little length assimilation occurs (Brigell \& Uhlarik, 1979). On the other hand, for the $90^{\circ}$ orientation, the contextual line would be almost completely within the attentive field specified by Jordan and Schiano (see Figure 4). The significant assimilation at the $45^{\circ}$ orientation could be due to the configuration's being a variant of the Ponzo illusion, or to the model being incorrect in specifying the boundary and shape of the attentive field.

Combining the results of the two experiments leads to the conclusion that length assimilation occurred at all orientations tested as long as the contextual and test lines were within the attentive field and they did not intersect. In terms of the model, we propose that the representations of the length of stimuli that can be sampled from within the attentive field are perceptually pooled (with assimilation the result) and that the representations of the length of these stimuli are orientation-free.

The conclusion that the combined results of Experiments 1 and 2 indicated a length distortion independent of orientation allowed a decision to be made on the nature of the relationship between length and orientation in the visual system. Relationship E (partial overlap of length and orientation information) is a good description of this relationship. The relationship implies at least three kinds of psychophysical effects, all of which have now been demonstrated-a length distortion independent of orientation, an orientation distortion independent of length (Wenderoth \& Johnson, 1984), and a length-dependent orientation distortion (Tyler \& Nakayama, 1984; Wenderoth et al., 1986). The demonstration of the orientationcontingent length distortion (Experiment 4, 60-sec condition) completed the set of effects implied by relationship E.

\section{Simultaneous Length Contrast}

Experiment 3 examined the orientation specificity of the simultaneous length-contrast effect first reported by Jordan and Schiano (1986). The data for the $0^{\circ}$ condition (parallel lines) replicated the reversal from length assimilation to length contrast with large spatial separation reported by Jordan and Schiano. Importantly, there was also significant length contrast at $12^{\circ}, 45^{\circ}$, and $90^{\circ}$ orien- 
tations of the contextual line, and the magnitude of this contrast did not differ from the $0^{\circ}$ condition. Thus, simultaneous length contrast does not seem to depend on the orientation of the contextual line relative to that of the test line. In terms of the Jordan and Schiano model, when the contextual stimulus is outside of the attentive field (due to a large spatial separation), it cannot be sampled at the same time as the test stimulus. In this case, contextual and test stimuli are sampled sequentially, with the initially sampled contextual stimulus being stored for differentiation from the subsequently sampled test stimulus, resulting in contrast. The data of Experiment 3 indicate that these stored representations of line length outside of the attentive field are orientation-free. Finally, the data are also supportive of relationship E (partial and mutual overlap) as descriptive of the coding of orientation and length within the visual system.

\section{Sequential Length Contrast}

Experiment 4 examined the orientation specificity of sequential length contrast (length aftereffect). A contextual line was inspected for either 5 or $60 \mathrm{sec}$ prior to test-line judgment. For the 5-sec condition, the obtained length contrast did not seem to be specific to the orientation of the contextual line; there was significant length contrast at all four orientations, and there were no differences in aftereffect magnitude across orientation. However, in the 60-sec condition, there was length contrast at all orientations, but it was greater for the $0^{\circ}$ (parallel-lines) condition than for the other conditions.

The 5-sec condition of Experiment 4 resulted in an amount of exposure to contextual and test elements that was similar to that of the simultaneous-presentation condition of Experiment 3. The essential difference between the two experiments was the mode of presentation (simultaneous or sequential) of contextual and test lines. That the results of the two conditions were so similar (i.e., length contrast was observed, and it was not specific to contextual-line orientation) supports the claim of Girgus and Coren (1982) that spatial and temporal separation of contextual and test elements are functionally equivalent in their ability to produce contrast. Additionally, the results for the two conditions lead to the conclusion that the representation of length outside of the attentive field that is stored is orientation-free. Finally, the observed orientation specificity in the 60 -sec inspection condition of Experiment 4 indicates that orientation specificity of length coding outside of the attentive field develops sometime between 5 and $60 \mathrm{sec}$ of inspection.

\section{Conclusions}

The results of the present experiments, combined with those of Tyler and Nakayama (1984), Wenderoth and Johnson (1984), and Wenderoth et al. (1986), indicate that there is partial overlap of length and orientation coding in the visual system. This conclusion is not surprising in that similar conclusions can be reached for color and spatial-frequency coding, as well as for orientation and spatial-frequency coding. In the case of color and spatial frequency, Virsu and Haapasalo (1973) reported a series of experiments demonstrating (1) color-contingent spatialfrequency contrast, (2) spatial-frequency contrast that did not depend on the color of the inducing and test stimuli, and (3) a color-contrast effect that did not initially depend on the spatial frequency of the inducing and test stimuli, but developed spatial frequency specificity as testing proceeded (note the similarity of this result to the finding of the present Experiment 4, in which orientation specificity of length contrast developed between 5 and $60 \mathrm{sec}$ of inspection of the contextual line). These three findings indicate that relationship E (partial overlap) describes the coding of color and spatial-frequency information.

The conclusion that orientation and spatial-frequency coding also partially overlap is based on the consideration of several experiments. First, Georgeson (1973) reported that the magnitude of simultaneous orientation contrast (the tilt illusion) was contingent on the spatial frequency of inducing and test stimuli. Additionally, Ware and Mitchell (1974) reported that the magnitude of sequential orientation contrast (the tilt aftereffect) was contingent on spatial frequency. Conversely, Campbell and Maffei (1971) and Parker (1972) reported that the magnitude of orientation contrast was unaffected by the spatial frequency of inducing and test stimuli. Finally, Heeley (1979) reported that spatial-frequency contrast could be induced by gratings oriented at either $0^{\circ}$ or $90^{\circ}$ relative to a test grating. Taken together, these experiments indicate both independence and overlap of orientation and spatial-frequency information, and thus support relationship $E$ as descriptive of orientation and spatial-frequency coding.

The perceptual consequences of this union relationship for the various parameters described so far were discussed by Virsu and Haapasalo (1973). According to these authors, when information for color and spatial frequency (for example) partially overlap, the resulting union channel can be "activated by stimuli whose colour or spatial frequency or both are correct" (p. 33). Furthermore, this seemingly general property of partial and mutual overlap of the coding of two parameters of form allows for both efficient integration and differentiation across these parameters. Thus, in the context of the present paper, we can perceive the length and orientation of a stimulus at once (integration) or we can perceive orientation or length independently (differentiation). Importantly, the partial overlap allows us to integrate or differentiate across dimensions at the same time (Virsu \& Haapasalo).

The partial independence of length and orientation information implies the need to postulate separate lengthcoding channels in the visual system. In fact, such channels have been proposed on the basis of psychophysical data. For example, Nakayama and Roberts (1972) reported that the contrast threshold of a moving grating was selectively elevated by adapting gratings; a short grating produced threshold elevation in a short test stimulus, but a long grating did not produce this elevation. Addi- 
tionally, Burton and Ruddock (1978) reported that contrast threshold elevation for test gratings was selective to the length of adapting gratings up to a point. When adapting-grating length was less than three times the individual bar width of the gratings, there was lengthselective threshold elevation; otherwise, threshold elevation was not specific to adapting-grating length. Although the results of Nakayama and Roberts (1972), Burton and Ruddock (1978), and the present experiments are suggestive of length-coding channels, the postulation of these channels is by no means necessary. It could be, as Wright (1982) and Ejima and Takahashi (1985) have argued, that length coding is accomplished within spatial-frequency channels; that is, that length coding is a subset of spatial frequency (compare with relationship D in Figure 1, in which length coding is represented as a subset of orientation coding). This possibility, of course, is not addressed by the present results, but it could be tested in a series of experiments in which the spatial-frequency specificity of length assimilation and contrast was assessed. These experiments are currently under way in our laboratory.

\section{REFERENCES}

APPELle, S. (1972). Perception and discrimination as a function of stimulus orientation: The "oblique effect" in man and animals. Psychological Bulletin, 78, 266-278.

AttNeave, F., Olson, R. (1967). Discriminability of stimuli varying in physical and retinal orientation. Journal of Experimental Psychology, 74, 149-157.

BrigelL, M., UHLARIK, J. (1979). The relational determination of length illusions and aftereffects. Perception, 8, 187-197.

Burton, G. J., \& RudDock, K. H. (1978). Visual adaptation to patterns containing two-dimensional spatial structure. Vision Research, 18, 93-99.

Campbell, F. W., \& Maffei, L. (1971). The tilt aftereffect: A fresh look. Vision Research, 11, 833-840.

Coren, S., \& GiRgus, J. S. (1972). A comparison of five methods of illusion measurement. Behavior Research Methods \& Instrumentation, 4, 240-244.

Coren, S., \& Girgus, J. S. (1978). Seeing is deceiving: The psychology of visual illusions. Hillsdale, NJ: Erlbaum.

EJIMA, Y., \& TAKaHASHI, S. (1985). Effect of localized grating adaptation as a function of separation along the length axis between test and adaptation areas. Vision Research, 25, 1701-1707.

FisheR, G. H. (1968). Gradients of distortion seen in the context of the Ponzo illusion and other contours. Quarterly Journal of Experimental Psychology, 20, 212-217.

Georgeson, M. A. (1973). Spatial frequency selectivity of a visual tilt illusion. Nature, 245, 43-45.

Girgus, J. S., \& CoREN, S. (1982). Assimilation and contrast illusions: Differences in plasticity. Perception \& Psychophysics, 32, 555-561.
HeEley, D. W. (1979). A perceived spatial frequency shift at orienta tions orthogonal to adapting gratings. Vision Research, 19, 1229-1236

JoRDAN, K., \& RANDALL, J. (1987). The effects of framing ratio anc oblique length on Ponzo illusion magnitude. Perception \& Psycho physics, 41, 435-439.

JoRDAN, K., \& SChIANo, D. J. (1986). Serial processing and the paralle lines illusion: Length contrast through relative spatial separation o contours. Perception \& Psychophysics, 40, 384-390.

JoRDAN, K., \& UHLARIK, J. (1985). Assimilation and contrast of perceived length depend on temporal factors. Perception \& Psychophysics $37,447-454$.

JORDAN, K., \& UHLARIK, J. (1986). Length contrast and the Müller Lyer figure: Functional equivalence of temporal and spatial separa tion. Perception \& Psychophysics, 39, 267-274.

Magnussen, S., \& Kurtenbach, W. (1980). Linear summation of th tilt illusion and aftereffect. Vision Research, 20, 39-42.

NAKAYAMA, K., \& RoBERTS, D. J. (1972). Line length detectors in the human visual system: Evidence from selective adaptation. Visior Research, 12, 1709-1713.

PARKer, D. M. (1972). Contrast and size variables and the tilt aftereffect Quarterly Journal of Experimental Psychology, 24, 1-7.

Pressey, A. W. (1972). The assimilation theory of geometric illlusions An additional postulate. Perception \& Psychophysics, 11, 28-30.

Pressey, A. W., \& D Lollo, V. (1978). Effects of distance betwee standard and comparison lines on the Müller-Lyer illusion. Percep tion \& Psychophysics, 24, 415-419.

Pressey, A. W., \& MurRay, R. (1976). Further developments in th assimilation theory of geometric illusions: The adjacency principle Perception \& Psychophysics, 19, 536-544.

Pressey, A. W., \& SMITH, N. E. (1986). The effects of location, orien tation, and cumulation of boxes in the Baldwin illusion. Perceptio \& Psychophysics, 40, 344-350.

Pressey, A. W., \& Wilson, A. E. (1980). Assimilation theory and th Baldwin illusion. Italian Journal of Psychology, 7, 65-73.

Tyler, C. W., \& Nakayama, K. (1984). Size interactions in the per ception of orientation. In L. Spillmann \& B. R. Wooten (Eds.), Sen sory experience, adaptation, and perception (pp. 529-546). Hillsdale NJ: Erlbaum.

Virsu, V., \& HaApasalo, S. (1973). Relationships between channel for colours and spatial frequency in human vision. Perception, 2 $31-40$.

WARE, C., \& Mrtchell, D. E. (1974). The spatial selectivity of th tilt aftereffect. Vision Research, 14, 735-737.

Wenderoth, P., \& Johinson, M. (1984). The effects of angle-arm lengtl on judgments of angle magnitude and orientation contrast. Percep tion \& Psychophysics, 36, 538-544.

Wenderoth, P., O'Connor, T., \& Johnson, M. (1986). The tilt illu sion as a function of the relative and absolute lengths of test and in ducing lines. Perception \& Psychophysics, 39, 339-345.

WRIGHT, M. J. (1982). Contrast sensitivity and adaptation as a func tion of grating length. Vision Research, 22, 139-149.

(Manuscript received March 2, 1987; revision accepted for publication November 2, 1987.) 